\title{
Non-linear Schrödinger Equation and Modified Korteweg-de Vries Equation; Construction of Solutions in Terms of Scattering Data.
}

By

\author{
Shunichi TANAKA*
}

In [16], we have constructed the general solutions of the initial value problem for the Korteweg-de Vries $(\mathrm{KdV})$ equation in terms of the scattering data of a Schrödinger operator. The purpose of the present paper is to extend the construction to the non-linear Schrödinger equation

$$
i u_{t}+2^{-1} u_{x x}+|u|^{2} u=0
$$

and to the the modified $\mathrm{KdV}$ equation

$$
v_{t}+6 v^{2} v_{x}+v_{x x x}=0 \text {. }
$$

Gardner, Greene, Kruskal and Miura (GGKM) [4] have discovered that the solution of the $\mathrm{KdV}$ equation can be described in terms of the scattering data of one-dimensional Scrödinger operator. They also proposed that the formalism of inverse scattering theory gives a certain explicit realization of solutions. If the reflection coefficient identically vanishes, the fundamental integral equation of the inverse problem reduces to linear algebraic equations and the potential is expressed in closed form in terms of exponentials. GGKM have also recognized that solutions of the $\mathrm{KdV}$ equation associated with reflectionless potentials play an important role in the study of asymptotic behavior of general solutions. See [13] for detailed discussion of reflectionless solutions of the $\mathrm{KdV}$ equatino.

An analogue of the GGKM theory for (0.1) has been initiated by Zakharov and Shabat [19]. They found that solutions of (0.1) are de-

Communicated by H. Yoshizawa, June 27, 1973.

* Department of Mathematics, Osaka University, Osaka. 
scribed in terms of scattering data of the operator

$$
L_{u}=i\left[\begin{array}{rr}
1 & 0 \\
0 & -1
\end{array}\right] D-i\left[\begin{array}{ll}
0 & u \\
u^{*} & 0
\end{array}\right] \quad(D=d / d x)
$$

with a complex potential $u$. Subsequently Wadati [17] and the present author [14] have associated (0.2) also with the operator $L_{u}$. In [19] some part of scattering theory of $L_{u}$ has been described on formal basis. In particular a part of reflectionless potentials and solutions associated with them are constructed.

Scattering theory for $L_{u}$ is more complicated than that of the onedimensional Schrödinger operator given in Faddeev [3] in several points. To explain them let $a(\zeta)$ denote the wronskian of the right and left Jost solutions (See $\S \S 1$ and 2 for detail). $a(\zeta)$ is defined and continuous for $\operatorname{Im} \zeta \geqq 0$, analytic in $\operatorname{Im} \zeta>0$ and tend to 1 as $|\zeta| \rightarrow \infty$. In the case of the Schrödinger operator, $a(\zeta)$ does not vanish on the real line, the number of its zeros are finite and all zeros are simple. These do not necessarily hold in our case.

In Shabat [10], [11], where scattering theory of $L_{u}$ is discussed, potentials whose $a(\zeta)$ has no zeros in $\operatorname{Im} \zeta \geqq 0$ are considered in deriving the fundamental integral equation and consequently in solving the inverse problem. This assumption excludes all reflectionless potentials and is inconvenient for the study of equations (0.1) and (0.2).

We relax the condition and only assume that $a(\zeta)$ has no zeros on the real line. The number of its zeros are finite in consequence. Even under this simplifying assumption, the theory is rather complicated by the fact that $a(\zeta)$ has multiple zeros. The complication due to the presence of multiple zeros already occurs for the Schrödinger equation with a complex potential (Blashchak [2]).

In $\S 1$ properties of the Jost solutions of $L_{u}$ are discussed. Then in $\S 2$ scattering data are defined and their properties are described. In $\S 3$ we derive the fundamental integral equations. $\$ \S 4$ and 5 concern the inverse problem. In $\S 4$ the solvability of the fundamental equation is discussed. Then in $\S 5$ the inverse problem is formulated and solved.

In deriving the above results, methods developed for Schrödinger 
equations ([1], [8] for radial equations; [3] for one-dimensional equations; [2] for equations with complex potentials) have been used in modified form.

The following two sections concern the construction of solutions of $(0.1)(\S 6)$ and $(0.2)(\S 7)$ in terms of scattering data of $L_{u}$. Several preliminary results have been published before in [14], [15].

Throughout the paper integrations are taken over $(-\infty, \infty)$ unless explicitly indicated. For a complex number $c, c^{*}$ denotes its complex conjugate.

\section{$\S 1$. Jost Solutions and Their Properties}

For a complex-valued measurable function $u$, consider the operator $L_{u}$ defined by $(0.3)$ on the infinite interval $(-\infty, \infty)$.

For a two-dimensional vector $y^{t}\left(y_{1}, y_{2}\right)$, put

$$
y^{*}={ }^{t}\left(y_{2}^{*},-y_{1}^{*}\right)
$$

and

$$
|y|=\max \left(\left|y_{1}\right|,\left|y_{2}\right|\right)
$$

If $y=y(x)$ is a solution of

$$
L_{u} y=\zeta y, \quad \zeta=\xi+i \eta
$$

then

$$
L_{u} y^{*}=\zeta^{*} y^{*}
$$

holds.

If $y(x)$ and $z(x)$ are solutions of (1.1), then the wronskian

$$
[y, z]=y_{1} z_{2}-y_{2} z_{1}
$$

is constant.

Put

$$
\begin{aligned}
& \psi_{-}^{\circ}(x, \zeta)=t(1,0) \exp (-i \zeta x), \\
& \psi_{+}^{\circ}(x, \zeta)=t(0,1) \exp (i \zeta x) .
\end{aligned}
$$

They are solutions of (1.1) for $u \equiv 0$. 
To obtain solutions with prescribed behavior as $x \rightarrow \pm \infty$, put the expression

$$
\psi(x)=\alpha(x) \psi_{+}^{\circ}(x)+\beta(x) \psi_{-}^{\circ}(x)
$$

into (1.1). Then we have

$$
\begin{aligned}
& \alpha^{\prime}(x)=-u^{*}(x) \psi_{1}(x) \exp (-i \zeta x) \\
& \beta^{\prime}(x)=u(x) \psi_{2}(x) \exp (i \psi x)
\end{aligned}
$$

We assume that the potential $u$ is integrable and put

$$
\sigma_{ \pm}(x)= \pm \int_{x}^{ \pm \infty}|u(y)| d y
$$

Theorem 1.1. There exist unique solutions $\psi_{ \pm}$of (1.1) for $\operatorname{Im} \zeta \geqq 0$ such that

$$
\left|\psi_{ \pm}(x, \zeta)-\psi_{ \pm}^{\circ}(x, \zeta)\right| \leqq C \sigma_{ \pm}(x) \exp (\mp \eta x)
$$

as $x \rightarrow \pm \infty . \quad \psi_{ \pm}$are analytic in $\zeta$ in the upper half plane.

Proof. By (1.2) we have the integral equations for $\psi_{ \pm}$:

$$
\psi_{ \pm}(x, \zeta)-\psi_{ \pm}^{\circ}(x, \zeta)=\left[\begin{array}{c}
-\int_{x}^{ \pm \infty} u(y) \psi_{ \pm 2}(y, \zeta) \exp (i \zeta(y-x)) d y \\
\int_{x}^{ \pm \infty} u^{*}(y) \psi_{ \pm 1}(y, \zeta) \exp (i \zeta(x-y)) d y
\end{array}\right]
$$

These integral equations can be solved by successive approximation leading to the existence of the solutions and their estimates. Q.E.D.

The solutions $\psi_{ \pm}$will be called the Jost solutions.

We have also

Lemma 1.2. There exist unique solutions $e_{ \pm}(x, \zeta)$ of $(1.1)$ for $\operatorname{Im} \zeta>0$ such that

$$
\exp ( \pm i \zeta x)\left\{e_{ \pm}(x, \zeta)-\psi_{ \pm}^{\circ}(x, \zeta)\right\} \rightarrow 0, \quad x \rightarrow \pm \infty
$$

These solutions are analytic in $\zeta$.

Proof. In this case we solve $(1.2)$ by the requirement that $a(a)=0$ 
and $\beta(x) \rightarrow 1$ as $x \rightarrow \infty$. Then we have the integral equations for $e=e_{+}$:

$$
\begin{aligned}
& e_{1}(x, \zeta)-\exp (-i \zeta x)=-\int_{x}^{\infty} u(y) e_{2}(y, \zeta) \exp (i \zeta(y-x)) d y \\
& e_{2}(x, \zeta)=-\int_{a}^{x} u^{*}(y) e_{1}(y, \zeta) \exp (i \zeta(x-y)) d y
\end{aligned}
$$

Putting

$$
e(x, \zeta)=\exp (-i \zeta x) h(x, \zeta)
$$

these are turned into integral equations for $h$ which can be solved by successive approximation provided $\sigma_{+}(a)<1$. It is easy to show that $h-t(1,0) \rightarrow 0$ as $x \rightarrow \infty$.

Q.E.D.

Put

$$
\psi_{ \pm}(x, \zeta)=\exp ( \pm i \zeta x) h_{ \pm}(x, \zeta)
$$

Assuming the integral representations

$$
\begin{array}{ll}
(1.4+) & h_{+}(x, \zeta)={ }^{t}(0,1)+\int_{0}^{\infty} B_{+}(x, y) \exp (2 i \zeta y) d y, \\
\left(1.4_{-}\right) & h_{-}(x, \zeta)={ }^{t}(1,0)+\int_{-\infty}^{0} B_{-}(x, y) \exp (-2 i \zeta y) d y,
\end{array}
$$

we put these into the integral equations for the Jost solutions. Then we have

$$
\begin{aligned}
& B_{+1}(x, y)+\int_{x}^{x+y} u(z) B_{+2}(z, x+y-z) d z=-u(x+y), \\
& B_{+2}(x, y)-\int_{x}^{\infty} u^{*}(z) B_{+1}(z, y) d z=0
\end{aligned}
$$

and

$$
\begin{aligned}
& B_{-1}(x, y)-\int_{-\infty}^{x} u(z) B_{-2}(z, y) d z=0, \\
& B_{-2}(x, y)+\int_{x+y}^{x} u^{*}(z) B_{-1}(z, x+y-z) d z=-u^{*}(x+y) .
\end{aligned}
$$

The potential $u$ is reproduced by

$$
u(x)=-B_{+1}(x, 0)=-B_{-2}^{*}(x, 0)
$$


Let $u$ be bounded integrable and put

$$
m_{ \pm}(x)=\operatorname{ess} \sup _{ \pm y \geq \pm x}|u(y)| \text {. }
$$

Then the integral equations for the kernels $B_{ \pm}$just obtained can be solved by succesisve approximation and the solutions are estimated as

$$
\left|B_{ \pm}(x, y)\right| \leqq m_{ \pm}(x+y) \exp \left(\sigma_{ \pm}(x)\right)
$$

(see [10, pp. 167-168]).

Now we study how additional smoothness of $u$ improves that of $B=B_{+}$.

Lemma 1.3. Suppose that $u$ is $n$-times differentiable with integrable derivatives. Then $B^{(j, k)}=\partial^{j+k} B / \partial x^{j} \partial y^{k}$ exist for $j+k \leqq n$ and the estimate

$$
\begin{gathered}
\left|B_{1}^{(j, k)}(x, y)+u^{(j+k)}(x+y)\right|+\left|B_{2}^{(j, k)}(x, y)\right| \\
\leqq C \sum_{i=0}^{j+k-1} m_{i}(x+y), \quad 1 \leqq j+k \leqq n,
\end{gathered}
$$

where

$$
m_{i}(x)=\sup _{y \geqq x}\left|u^{(i)}(x)\right|
$$

hold.

Proof. By (1.6), $B_{2}$ is differentiable and

$$
B_{2 x}(x, y)=-u^{*}(x) B_{1}(x, y)
$$

holds. By (1.5) $B_{1}$ is differentiable with respect to $x$ and estimate for $B_{x}$ follows. Results for $B^{(j, 0)}$ follow by induction on $j$ using (1.5) and (1.8).

By (1.5) $B_{1}$ is differentiable with respect to $y$ and

$$
B_{1 x}(x, y)-B_{1 y}(x, y)=u(x) B_{2}(x, y)
$$

holds. $\quad B_{2 y}$ exists by (1.6) and the estimate for $B_{y}$ follows.

For general $B^{(j, k)}$ the assertion follows from double induction on $j+k$ and $k$.

Q.E.D.

If $m_{ \pm}( \pm x) \in L^{1}(a, \infty)$, we can define $\psi_{ \pm}$by $(1.3)$ and (1.4). $\psi_{ \pm}$coincide with the Jost solutions. So we have.

Theorem 1.4. Suppose that $u$ is bounded and $m_{ \pm}( \pm x)$ are in $L^{1}(a, \infty)$ 
for some $a$. Then there exist kernels $B_{ \pm}$estimated as (1.7) and Jost solutions are represented as (1.3), (1.4).

\section{$\S$ 2. Scattering Data and Their Properties}

Now let $\zeta=\xi$ be real. Then

$$
\left[\psi_{ \pm}, \psi_{ \pm}^{*}\right]=-1
$$

As $\psi_{+}$and $\psi_{+}^{*}$ are linearly independent solutions of $(1.1)$ for $\zeta=\xi$, we can expand $\psi$ - uniquely as

$$
\psi_{-}=a(\xi) \psi_{+}^{*}+b(\xi) \psi_{+} .
$$

Similarly

$$
\psi_{+}=c(\xi) \psi_{-}^{\#}+d(\xi), \psi_{-} .
$$

We have

$$
a(\xi)=\left[\psi_{-}, \psi_{+}\right], \quad b(\xi)=-\left[\psi_{-}, \psi_{+}^{\sharp}\right]
$$

and

$$
a(\xi)=-c(\xi), \quad b(\xi)=d(\xi)^{*}
$$

Putting (2.2) into (2.1) and using the relations (2.4), we have

$$
|a(\xi)|^{2}+|b(\xi)|^{2}=1
$$

Putting (1.3), (1.4) into (2.3), we have

$$
a(\zeta)=1+\int_{0}^{\infty} \Gamma(y) \exp (2 i \zeta y) d y
$$

where

(2.6) $\quad \Gamma(y)=B_{-1}(x,-y)+B_{+2}(x, y)$

$$
+\int_{0}^{y}\left[B_{-1}(x,-z) B_{+2}(x, y-z)-B_{-2}(x,-z) B_{+1}(x, y-z)\right] d z
$$

and

$$
b(\xi)=\int \Pi(y) \exp (-2 i \xi y) d y
$$


where

$$
\begin{aligned}
& \exp (2 i \xi x) \Pi(y)=B_{+1}^{*}(x, y)+B_{-2}(x, y) \\
& \quad+\int B_{-1}(x, z) B_{+1}^{*}(x, y-z) d z+\int B_{-2}(x, z) B_{+2}^{*}(x, y-z) d z
\end{aligned}
$$

(we have extended $B_{ \pm}(x, y)$ as 0 for $\pm y<0$ ).

Putting $x=0$, for example, we have the estimates:

$$
|\Pi(y)| \leqq C m_{ \pm}( \pm y), \quad \pm y>0
$$

and

$$
|\Gamma(y)| \leqq C\left(m_{-}\left(-2^{-1} y\right)+m_{+}\left(2^{-1} y\right)\right), \quad y>0
$$

$\Gamma$ and $\Pi$ are bounded integrable functions.

There are other expressions for $\Gamma$ and $\Pi$ which are sometimes more convenient.

Considering the limit for $x \rightarrow \infty$ in (2.1) and comparing the coefficients of $\exp ( \pm i \xi x)$, we have the integral representations

$$
\begin{aligned}
& a(\xi)=1+\int u(y) \psi_{-2}(y, \xi) \exp (i \xi y) d y \\
& b(\xi)=-\int u^{*}(y) \psi_{-1}(y, \xi) \exp (-i \xi y) d y
\end{aligned}
$$

Putting (1.3), (1.4) into these expressions and performing a formal change of the order of integrations, we obtain the expressions:

$$
\begin{aligned}
& \Pi(y)=-u(y)-\int_{y}^{\infty} u^{*}(x) B_{-1}(x, y-x) d x \\
& \Gamma(y)=\int u(x) B_{-2}(x,-y) d x
\end{aligned}
$$

For functions defined by (2.7) and (2.8) we have the estimates

$$
\left|\Pi\left(y^{\prime}\right)+u(y)\right| \leqq C \sigma_{+}(y) m_{-}(y)
$$

and

$$
|\Gamma(y)| \leqq C\left(m_{-}\left(-2^{-1} y\right)+\sigma_{+}\left(2^{-1} y\right)\right) .
$$

Thus we have 
Lemma 2.1. If $u$ satisfies the assumption of Theorem 1.4 and $(1+|x|) u$ is integrable, then expressions (2.7) and (2.8) hold.

Lemma 2.2. Let $u$ be in $S$, the space of $C^{\infty}$-functions which are rapidly decreasing together with all derivatives. Then $I$ is in $S . \quad \Gamma$ is $C^{\infty}$ and rapidly decreasing as $x \rightarrow \infty$ together with all derivatives.

Proof. By repeated differentiation of (2.7), (2.8) and by Lemma 1.3, we obtain similar estimates to (2.9) and (2.10) for each derivative. Q.E.D.

Let $\zeta_{0}$ be a zero of order $m$ of $a(\zeta)$ in the upper half plane. Because $\psi_{ \pm}$are linearly dependent at $\zeta=\zeta_{0}$, they are square integrable by their asymptotic property.

Theorem 2.3. There exists a chain of complex numbers $c^{+}=\left\{c_{0}^{+}, \ldots\right.$, $\left.c_{m-1}^{+}\right\}\left(c_{0} \neq 0\right)$ such that

$$
j^{1-1} d^{j} \psi_{-}\left(x, \zeta_{0}\right) / d \zeta^{j}=\sum_{\mu=0}^{j} \mu !^{-1} c_{j-\mu}^{+} d^{\mu} \psi_{+}\left(x, \zeta_{0}\right) / d \zeta^{\mu}, \quad 0 \leqq j \leqq m-1,
$$
hold.

Proof. Let $e_{+}(x, \zeta)$ be the solution of (1.1) defined in Lemma 1.2. Noting that $\left[\psi_{+}, e_{+}\right]=-1$, we expand $\psi_{-}$as

$$
\psi_{-}=c(\zeta) \psi_{+}+a(\zeta) e_{+}
$$

where $c(\zeta)=\left[e_{+}, \psi_{-}\right]$is analytic in the upper half plane.

Comparing the $j$-th coefficient of the Taylor expansion of (2.12) at $\zeta=\zeta_{0}$, we have $(2.11)$ where

$$
c^{+}=\mu !^{-1} d^{\mu} c\left(\zeta_{0}\right) / d \zeta^{\mu} .
$$

Obviously the chain of complex numbers $\boldsymbol{c}^{-}=\left\{\bar{c}_{0}^{-}, \ldots, \bar{c}_{m-1}^{-}\right\}$is uniquely determined such that

$$
\left[\begin{array}{ccccc}
c_{0}^{+} & 0 & \cdots \cdots & 0 \\
c_{1}^{+} & c_{0}^{+} & 0 & \cdots & 0 \\
& \cdots \cdots \cdots \cdots \cdots & \cdots & \cdots \\
c_{m-1}^{+} & c_{m-2}^{+} & \cdots \cdots & \cdots & c_{0}^{+}
\end{array}\right]\left[\begin{array}{ccccc}
c_{0}^{-} & 0 & \cdots \cdots & 0 \\
c_{1}^{-} & c_{0}^{-} & 0 & \cdots & 0 \\
& \cdots \cdots \cdots \cdots \cdots \cdots & \\
c_{m-1}^{-} & c_{m-2}^{-} & \cdots \cdots & \cdots & c_{0}^{-}
\end{array}\right]=I .
$$

The chains $\boldsymbol{c}^{ \pm}$are called the normalization chains associated with the zero 
$\zeta_{0}$ of $a(\zeta)$.

We now assume that

$$
a(\xi) \neq 0
$$

for any real $\xi$. As the integral representation (2.5) holds with the integrable kernel $\Gamma, a(\zeta) \rightarrow 1$ as $|\xi| \rightarrow \infty$. By this property and (2.14), $a(\zeta)$ has only a finite number of zeros in the upper half plane. We denote them by $\zeta_{j}(j=1, \ldots, N)$ and their multiplicity by $m(j)$. Denote by $\boldsymbol{c}_{j}^{ \pm}$the chain associated with the zero $\zeta_{j}$ by Theorem 2.3 .

Put

$$
r_{+}(\xi)=b(\xi) a(\xi)^{-1}, \quad r_{-}(\xi)=d(\xi) c(\xi)^{-1}
$$

$r_{ \pm}(\xi)$ are called the reflection coefficients. The collections

$$
s_{ \pm}=\left\{r_{ \pm}(\xi), \zeta_{j}, \boldsymbol{c}_{j}^{ \pm}\right\}
$$

are called the scattering data of the operator $L_{u}$ or the potential $u$.

An extension of the Wiener-Lévy theorem [6, Lemma 1.2] gives

Lemma 2.4. Let $a(\zeta)$ be defined by

$$
a(\zeta)=1+\int_{0}^{\infty} \Gamma(y) \exp (2 i \zeta y) d y, \quad \Gamma \in L^{1}(0, \infty),
$$

and let $a(\xi) \neq 0$ for any real $\xi$. Let $\zeta_{j}(j=1, \ldots, N)$ be the zeors of order $m(j)$ of $a(\zeta)$ in the upper half plane. Then $a(\zeta)$ has the representation

$$
a(\zeta)=\prod_{j=1}^{N}\left(\zeta-\zeta_{j} / \zeta-\zeta_{j}^{*}\right)^{m(j)} \exp \left(\int_{0}^{\infty} \Gamma_{1}(t) \exp (2 i \zeta t) d t\right)
$$

where $\Gamma_{1}$ is in $L^{1}(0, \infty)$.

Putting $\zeta=\xi$ in (2.15), we have

$$
\log |a(\xi)|=\operatorname{Re} \int_{0}^{\infty} \Gamma_{1}(t) \exp (2 i \xi t) d t
$$

\section{§3. Derivation of the Fundamental Integral Equations}

In this section we derive integral equations which connect the kernels $B_{ \pm}$with the scattering data. 
As the coefficient $b(\xi)$ is the Fourier transform of a bounded $L^{1}$. function, it is a bounded $L^{2}$-function and tends to zero as $\xi \rightarrow \pm \infty$. So $r_{ \pm}(\xi)$ are also bounded $L^{2}$-functions and tend to zero as $\xi \rightarrow \pm \infty$. Put

$$
F_{ \pm}(x)=\pi^{-1} \int r_{ \pm}(\xi) \exp ( \pm 2 i \xi x) d x
$$

as $L^{2}$-Fourier transforms.

Now on the identity

$$
a^{-1}(\xi) h_{-}(x, \xi)-{ }^{t}(1,0)=\left\{h_{+}(x, \xi)-{ }^{t}(0,1)\right\}^{\#}+r_{+}(\xi) \exp (2 i \xi x) h_{+}(x, \xi)
$$

multiply by $\pi^{-1} \exp (2 i \xi y)$ and integrate over $(-\infty, \infty)$. By the convolution theorem, the right-hand side gives

$$
B_{+}^{*}(x, y)+\left[\begin{array}{c}
0 \\
F_{+}(x+y)
\end{array}\right]+\int_{0}^{\infty} F_{+}(x+y+z) B_{+}(x, z) d z .
$$

By Jordan's lemma $[12, \S 60]$, we have

$$
\begin{gathered}
\pi^{-1} \int\left\{a^{-1}(\xi) h_{-}(x, \xi)-{ }^{t}(1,0)\right\} \exp (2 i \xi y) d \xi \\
=2 i \sum_{j=1}^{N} R\left(\zeta_{j}\right)
\end{gathered}
$$

where $R\left(\zeta_{0}\right)$ is the residue of

$$
a^{-1}(\zeta) h_{-}(x, \zeta) \exp (2 i \zeta y)
$$

at a zero $\zeta_{0}$ of $a(\zeta)$.

Next we express $R\left(\zeta_{0}\right)$ in terms of the kernel $B_{+}$. We have

$$
R\left(\zeta_{0}\right)=(m-1) !^{-1}(d / d \zeta)^{m-1} a_{0}(\zeta)^{-1} h_{-}(x, \zeta) \exp (2 i \zeta y) \quad\left(\zeta=\zeta_{0}\right)
$$

where

$$
a_{0}(\zeta)=a(\zeta)\left(\zeta-\zeta_{0}\right)^{-m}
$$

By the relation (2.11),

$$
\begin{aligned}
R\left(\zeta_{0}\right)=\sum_{\mu=0}^{m-1} \mu !^{-1} c_{m-\mu-1}^{+}(d \mid d \zeta)^{\mu} a_{0}(\zeta)^{-1} h_{+}(x, \zeta) & \\
& \times \exp (2 i \zeta(x+y)) \quad\left(\zeta=\zeta_{0}\right)
\end{aligned}
$$

Put 


$$
f_{ \pm}(x)=\mp 2 i \sum_{\mu=0}^{m-1} \mu !^{-1} c_{m-\mu-1}^{ \pm}(d / d \zeta)^{\mu} a_{0}(\zeta)^{-1} \exp ( \pm 2 i \zeta x) \quad\left(\zeta=\zeta_{0}\right) .
$$

$f_{ \pm}(x)$ are of the form

$$
f_{ \pm}(x)=p_{ \pm}(x) \exp \left( \pm 2 i \zeta_{0} x\right)
$$

where $p_{ \pm}(x)$ are polynomials of order $m-1$.

$R\left(\zeta_{0}\right)$ is now expressed as

$$
R\left(\zeta_{0}\right)=-(2 i)^{-1}\left\{\left[\begin{array}{c}
0 \\
f_{+}(x+y)
\end{array}\right]+\int_{0}^{\infty} f_{+}(x+y+z) B_{+}(x, z) d z\right\} .
$$

Coming back to the derivation of integral equations for the kernels $B_{ \pm}$, let $f_{ \pm j}(x)$ be functions defined by (3.4) at the zeros $\zeta_{j}$ of $a(\zeta)$. Put

$$
\Omega_{ \pm}(x)=F_{ \pm}(x)+\sum_{j=1}^{N} f_{ \pm j}(x)
$$

Putting (3.5) into the equality $(3.1)=(3.2)$, we have

$$
(3.6+) \quad B_{+}^{\sharp}(x, y)+\int_{0}^{\infty} \Omega_{+}(x+y+z) B_{+}(x, z) d z+\left[\begin{array}{c}
0 \\
\Omega_{+}(x+y)
\end{array}\right]=0 .
$$

Analogously

(3.6-) $\quad B_{-}^{*}(x, y)+\int_{-\infty}^{0} \Omega_{-}(x+y+z) B_{-}(x, z) d z+\left[\begin{array}{c}\Omega_{-}(x+y) \\ 0\end{array}\right]=0$

holds.

These analogues of the Marchenko equation are first introduced in [19] under the assumption that all zeros of $a(\zeta)$ are simple.

\section{$\S 4$. Solvability of the Fundamental Integral Equations}

In this section we discuss the solvability of (3.6) as an integral equation for $B_{+}$.

Given a function $\Omega(x)$ belonging to $L^{1}(a, \infty)$ for any $a$, we define the integral operator $\Omega_{x}$ by

$$
\Omega_{x} \varphi(y)=\int_{0}^{\infty} \Omega(x+y+z) \varphi(z) d z
$$


as an operator on $L^{1}(0, \infty) . \quad \Omega_{x}$ is a completely continuous operator (see [1, pp. 70-72]).

We consider the solvability of

$$
\varphi^{\sharp}+\Omega_{x} \varphi=\psi
$$

which is a slight generalization of $(3.6+)$.

Eliminating $\psi_{1}$ or $\psi_{2}$ we have

$$
\begin{aligned}
& \left(I+\Omega_{x}^{*} \Omega_{x}\right) \varphi_{1}=-\psi_{2}^{*}-\Omega_{x}^{*} \psi_{1}, \\
& \left(I+\Omega_{x}^{*} \Omega_{x}\right) \varphi_{2}=\psi_{1}^{*}+\Omega_{x}^{*} \psi_{2} .
\end{aligned}
$$

If one of these equations is solved, the other component can be determined from the original equation (4.1).

Lemma 4.1. Suppose $\Omega(x)$ is bounded integrable in $(a, \infty)$ for any a. Then

(a) the operator $\left(I+\Omega_{x}^{*} \Omega_{x}\right)$ has bounded inverse,

(b) $\left(I+\Omega_{x}^{*} \Omega_{x}\right)^{-1}$ is continuous in $x$ and bounded as $x \rightarrow \infty$ in the operator norm (topology),

(c) the system (4.1) is uniquly solvable for any $\psi$ and the estimate

$$
\|\varphi\| \leqq C(x)\|\psi\|
$$

holds for the solution. $C(x)$ denotes a certain non-increasing function.

Proof. Suppose $\varphi$ is a solution of

$$
\left(I+\Omega_{x}^{*} \Omega_{x}\right) \varphi=0
$$

in $L^{1}(0, \infty)$. By the properties of $\Omega$ and (4.4), the boundedness of $\varphi$ follows. So $\varphi$ belongs to $L^{2}$. In $L^{2}$ the operator $\Omega_{x}^{*}$ is equal to $\left(\Omega_{x}\right)^{*}$, the adjoint of $\Omega_{x}$, and therefore $\varphi$ should be zero. By the complete continuity of $\Omega_{x}$, the assertion (a) follows.

In the operator norm $\Omega_{x}$ is continuous in $x$ and tends to zero as $x \rightarrow \infty$. The assertion (b) follows. (c) is obtained by applying (b) to (4.2) and (4.3).

Q.E.D.

Let $r(\xi)$ be a square-integrable function. Let $\zeta_{1}, \ldots, \zeta_{N}$ denote points 
in the upper half plane. For each $j$, let $m(j)$ be natural numbers and $p_{j}(x)$ be polynomials of order $m(j)-1$. Put

$$
f_{j}(x)=p_{j}(x) \exp \left(2 i \zeta_{j} x\right)
$$

Put

$$
F(x)=\pi^{-1} \int r(\xi) \exp (2 i \xi x) d \xi
$$

and

$$
\Omega(x)=F(x)+\sum_{j=1}^{N} f_{j}(x)
$$

Put

$$
\sigma_{j}(x)=\int_{x}^{\infty}\left|\Omega^{(j)}(y)\right| d y
$$

if the right hand side exists.

By Lemma 4.1, we get

Theorem 4.2. If $\Omega(x)$ is bounded integrable in $(a, \infty)$ for any $a$, there exists a unique solution $B(x, y)$, integrable in $y$, of

$$
B^{*}(x, y)+\int_{0}^{\infty} \Omega(x+y+z) B(x, z) d z+\left[\begin{array}{c}
0 \\
\Omega(x+y)
\end{array}\right]=0 .
$$

The estimates

$$
\int_{0}^{\infty}|B(x, y)| d y \leqq C(x)
$$

and

$$
|B(x, y)| \leqq C(x) \sup _{z \geqq x+y}|\Omega(z)|
$$

hold.

Returning to the general situation, we obtain

Lemma 4.3. Suppose that $\Omega$ is absolutely continuous and $\Omega, \Omega^{\prime}$ are in $L^{1}(a, \infty)$ for any $a$. Let $\psi_{x}$ be differentiable in $x$ and let $\varphi_{x}$ be the solution of (4.1) for $\psi=\psi_{x}$ i.e.

$$
\varphi_{x}^{\sharp}+\Omega_{x} \varphi_{x}=\psi_{x} .
$$


Then $\varphi_{x}$ is differentiable in $x$ and

$$
\varphi_{x}^{\prime \#}+\Omega_{x} \varphi_{x}^{\prime}=\psi_{x}^{\prime}-\Omega_{x}^{\prime} \varphi_{x}
$$

holds.

Proof. For the difference coefficient

$$
\varphi_{x, h}=h^{-1}\left(\varphi_{x+h}-\varphi_{x}\right)
$$

we have the equation

$$
\varphi_{x, h}^{\#}+\Omega_{x} \varphi_{x, h}=\psi_{x, h}-h^{-1}\left(\Omega_{x+h}-\Omega_{x}\right) \varphi_{x+h} .
$$

The right hand side converges to

$$
\psi_{x}^{\prime}-\Omega_{x}^{\prime} \varphi_{x}
$$

as $h \rightarrow 0$. Eliminating each component of $\varphi_{x, h}$ from (4.10) and applying Lemma 4.1 (a), we conclude that $\varphi_{x, h}$ converges to $\varphi_{x}^{\prime}$.

Q.E.D.

Under the same assumption on $\Omega, B$ defined by (4.5) is differentiable in $y$ and the estimates

$$
\int_{0}^{\infty}|B y(x, y)| d y \leqq C(x)
$$

and

$$
\mid B_{y}(x, y)+\left[\begin{array}{c}
0 \\
\Omega^{\prime}(x+y)
\end{array}\right] \leqq C(x) \sigma_{1}(x+y)
$$

hold. A similar result for the $x$-derivative can be obtained by Lemma 4.3. In fact $B(x, y)$ is differentiable in $x$ in the $L^{1}$ sense and the estimate

$$
\int_{0}^{\infty}\left|B_{x}(x, y)\right| d y \leqq C(x)
$$

holds. Then it is easy to see that the derivative

$$
(d / d x) \int_{0}^{\infty} \Omega(x+y+z) B(x, z) d z
$$

exists in the ordinary sense and we can change the order of integration and differentiation. $B_{x}$ also satisfies the inequality (4.11). 
Put

$$
\psi(x, \zeta)=\exp (i \zeta x)\left\{t(0,1)+\int_{0}^{\infty} B(x, y) \exp (2 i \zeta y) d y\right\}
$$

Then $\psi$ is differentiable in $x$ by the results just described.

Theorem 4.4. If $\Omega$ is absolutely continuous and $\Omega, \Omega^{\prime}$ are in $L^{1}(a, \infty)$ for any $a$, then $\psi$ defined above satisfies

$$
L_{u} \psi=\zeta \psi
$$

for

$$
u(x)=-B_{1}(x, 0)
$$

Proof. Put

$$
\begin{aligned}
& C_{1}(x, y)=-B_{2 x}(x, y)-u^{*}(x) B_{1}(x, y), \\
& C_{2}(x, y)=B_{1 x}(x, y)-B_{1 y}(x, y)-u(x) B_{2}(x, y) .
\end{aligned}
$$

Then by direct calculation we have

$$
C^{\sharp}(x, y)+\int_{0}^{\infty} \Omega(x+y+z) C(x, z) d z=0 .
$$

So $C=0$ follows. Once $C=0$ is established, the assertion follows by straightfoward calculation.

Q.E.D.

By induction based on Lemma 4.3, we have

Lemma 4.5. Suppose that $\Omega^{(j)}, j \leqq n$ exist and belong to $L^{1}(a, \infty)$ for any $a$. Then $B^{(j, k)}(x, y), j+k \leqq n$, exist and the estimates

$$
\int_{0}^{\infty}\left|B^{(j, k)}(x, y)\right| d y \leqq C(x)
$$

and

$$
\mid B^{(j, k)}(x, y)+\left[\begin{array}{c}
0 \\
\Omega^{(j+k)}(x+y)
\end{array}\right] \leqq C(x) \Sigma_{i=0}^{j+k} \sigma_{i}(x+y)
$$

hold. 


\section{$\S 5$. Solution of the Inverse Problem}

Let $r(\xi)$ be a square integrable continuous function. Assume that there exists $a(\zeta)$ which has the properties assumed in Lemma 2.4 and is related to $r(\xi)$ by

$$
|a(\xi)|^{2}=\left(1+|r(\xi)|^{2}\right)^{-1} .
$$

By (2.15), $\Gamma_{1}(t)$ in Lemma 2.4 is uniquely determined by this relation.

Put $r_{+}(\xi)=r(\xi)$ and

$$
r_{-}(\xi)=-a(\xi)^{-1} a^{*}(\xi) r^{*}(\xi) .
$$

Define $F_{ \pm}(x)$ by the formula

$$
F_{ \pm}(x)=\pi^{-1} \int r_{ \pm}(\xi) \exp ( \pm 2 i \xi x) d \xi
$$

Assume that $F_{ \pm}(x)$ are absolutely integrable and $F_{ \pm}( \pm x), F_{ \pm}^{\prime}( \pm x)$ are in $L^{1}(a, \infty)$ for any $a$.

In the later sections, we will be concerned in the case where $r(\xi)$ is in $S$. Then $\log \left(1+|r(\xi)|^{2}\right)$ is also in $S$. So (2.16) determines $\Gamma_{1}(t)$ as the restriction to $(0, \infty)$ of an element in $S . a(\zeta)$ defined by $(2.15)$ has the required properties. $\quad r_{ \pm}$are in $\mathrm{S}$ and so are $F_{ \pm}$.

To each zero $\zeta_{j}$ of order $m(j)$ of $a(\zeta)$, we associate the chains of complex numbers

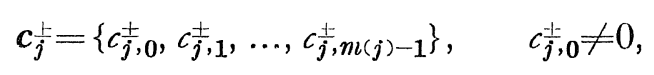

each connected by the relation (2.13).

Define $f_{ \pm j}(x)$ by the formulas (3.4) and put

$$
\Omega_{ \pm}(x)=F_{ \pm}(x)+\sum_{j=1}^{N} f_{ \pm j}(x) .
$$

Consider the fundamental integral equations (3.6) and let $B_{ \pm}(\lambda, y)$ be their solutions. Define $h_{ \pm}(x, \zeta)$ by $(1.4)$ and then $\psi_{ \pm}(x, \zeta)$ by (1.3). Put

$$
u_{+}(x)=-B_{+1}(x, 0) \quad u_{-}(x)=-B_{-2}^{*}(x, 0) .
$$

Then, by Theorem $4.6, \psi_{ \pm}$satisfy (1.1) with $u=u_{ \pm}$respectively. For $B_{ \pm}$, the estimates formulated in Theorem 4.2 hold. 
We show that under certain additional conditions $u_{ \pm}(x)$ coincide. It follows immediately, once the relation

$$
a(\xi)^{-1} \psi_{-}(x, \xi)=\psi_{+}^{*}(x, \xi)+r_{+}(\xi) \psi_{+}(x, \xi)
$$

is established.

To show that (5.3) holds, put

$$
g(x, \xi)=h_{+}^{\sharp}(x, \xi)+r_{+}(\xi) \exp (2 i \xi x) h_{+}(x, \xi)
$$

and

$$
\begin{aligned}
G(x, y) & =\pi^{-1} \int\left(g(x, \xi)-{ }^{t}(1,0)\right) \exp (2 i \xi y) d \xi \\
& =B_{+}^{\sharp}(x, y)+\int_{0}^{\infty} F_{+}(x+y+z) B_{+}(x, z) d z+\left[\begin{array}{c}
0 \\
F_{+}(x+y)
\end{array}\right] .
\end{aligned}
$$

Then by the fundamental equation, we have

$$
G(x, y)=-\sum_{j=1}^{N}\left\{\left[\begin{array}{c}
0 \\
f_{+j}(x+y)
\end{array}\right]+\int_{0}^{\infty} f_{+j}(x+y+z) B_{+}(x, z) d z\right\}
$$

for $y>0$.

$g(x, \xi)$ which are defined for real $\xi$ can be analytically continued to the upper half plane. To show this we need

Lemma 5.1. Put

$$
\begin{array}{cl}
f(x)=p(x) \exp \left(2 i \zeta_{0} x\right) & x>0, \\
0 & x<0,
\end{array}
$$

where $p(x)$ is a polynomial of order $m-1$. Then we have, for $x+y>0$,

$$
\left[\begin{array}{c}
0 \\
f(x+y)
\end{array}\right]+\int_{0}^{\infty} f(x+y+z) B_{+}(x, z) d z=p(x, y) \exp \left(2 i \zeta_{0} y\right)
$$

where each component of $p(x, y)$ is defined for $x+y>0$ and is a polynomial of order $m-1$ in $y$.

Proof. $f(x)$ is written as a Fourier transform:

$$
f(x)=\pi^{-1} \int q(\xi) \exp (2 i \xi x) d \xi
$$


where $q(\xi)$ is a polynomial of order $m$ in $\left(\xi-\zeta_{0}\right)^{-1}$ without constant term. Then for $x+y>0$, the left hand side of (5.4) is equal to

$$
\begin{aligned}
& \pi^{-1} \int q(\xi) \psi_{+}(x, \xi) \exp (i \xi x+2 i \xi y) d \xi \\
& =2 i \operatorname{Res} \zeta_{=}\left[q(\zeta) \psi_{+}(x, \zeta) \exp (i \zeta x+2 i \zeta y)\right] \\
& =2 i(m-1) !^{-1}(d \mid d \zeta)^{m-1} q(\zeta)\left(\zeta-\zeta_{0}\right)^{m} \psi_{+}(x, \zeta) \exp (i \zeta x+2 i \zeta y) \\
& \left(\zeta=\zeta_{0}\right) .
\end{aligned}
$$

The last term can be written as asserted.

Q.E.D.

We have further

$$
p(x, y) \exp \left(2 i \zeta_{0} y\right)=\pi^{-1} \int q(x, \xi) \exp (2 i \xi y) d \xi, \quad y>0
$$

where $q(x, \zeta)$ is a polynomial of order $m$ in $\left(\zeta-\zeta_{0}\right)^{-1}$ without constant term.

Denote by $q_{j}(x, \zeta)$ the functions connected with $f_{+j}(x)$ by the above construction.

Put

$$
g_{1}(x, \xi)=g(x, \xi)-{ }^{t}(1,0)+\sum_{j=1}^{N} q_{j}(x, \xi)
$$

Then, for $x+y, y>0$, we have

$$
\int g_{1}(x, \xi) \exp (2 i \xi y) d \xi=0
$$

So $g_{1}(x, \xi)$ has analytical continuation as a holmorphic function into the upper half plane. $g(x, \xi)$ has analytical continuation as a meromorphic function into the upper half plane. Its poles are exhausted by $\zeta_{1}, \ldots, \zeta_{N}$. $\zeta_{j}$ are poles of order $m(j)$.

Denote by $R_{j}$ the residue of

$$
g(x, \zeta) \exp (2 i \zeta y), \quad y>0
$$

at $\zeta_{j}$, i.e. the residue of

$$
-q_{j}(x, \zeta) \exp (2 i \zeta y)
$$

at $\zeta_{j}$. It is expressed as 


$$
R_{j}=-(2 \pi i)^{-1} \int q_{j}(x, \xi) \exp (2 i \xi y) d \xi
$$

By (5.4) and (5.5), we have, for $x+y, y>0$,

$$
R_{j}=-(2 i)^{-1}\left\{\left[\begin{array}{c}
0 \\
f_{j}(x+y)
\end{array}\right]+\int_{0}^{\infty} f_{j}(x+y+z) B_{+}(x, z) d z\right\} .
$$

By the definition of $f_{j}$, we have

$$
R_{j}=\sum_{\mu=0}^{m(j)-1} \mu !^{-1} c_{j, m(j)-1-\mu}(d / d \zeta)^{\mu} a_{j}(\zeta) \psi_{+}(x, \zeta) \exp (i \zeta x+2 i \zeta y) \quad\left(\zeta=\zeta_{j}\right)
$$

where

$$
a_{j}(\zeta)=\left(\zeta-\zeta_{j}\right)^{-m(j)} a(\zeta)
$$

Put

$$
h(x, \zeta)=a(\zeta) g(x, \zeta)
$$

and

$$
\psi(x, \zeta)=h(x, \zeta) \exp (-i \zeta x)
$$

Then $\psi(x, \zeta)$ is holomorphic in the upper half plane. $R_{j}$ is also equal to the residue of

$$
a(\zeta)^{-1} \psi(x, \zeta) \exp (i \zeta x+2 i \zeta y)
$$

at $\zeta_{j}$ and is therefore expressed as

$$
R_{j}=(m-1) !^{-1}(d / d \zeta)^{m-1} a_{j}(\zeta)^{-1} \psi(x, \zeta) \exp (i \zeta x+2 i \zeta y), \quad\left(\zeta=\zeta_{j}\right)
$$

Comparison of (5.7), (5.10) and an elementary argument lead to

$$
\begin{aligned}
k !^{-1}(d \mid d \zeta)^{k} \psi\left(x, \zeta_{j}\right)=\sum_{\mu=0}^{k} \mu !^{-1} c_{j, k}^{+}-\mu(d \mid d \zeta)^{\mu} \psi_{+}\left(x, \zeta_{j}\right), & \\
& 0 \leqq k \leqq m(j)-1 .
\end{aligned}
$$

Theorem 5.2. Let $r(\xi)=O\left(\xi^{-1}\right), \xi \rightarrow \pm \infty$, and also let the conditions formulated in the beginning of this section be satisfied. Then $h(x, \zeta)$ defined by (5.8) is represented as

$$
h(x, \zeta)=t(1,0)+\int_{-\infty}^{0} C(x, y) \exp (-2 i \zeta y) d y, \quad x>0
$$


$C(x, y)$ being square integrable in $y . \quad C(x, y)(x>0)$ is the solution of the fundamental equation

$$
C(x, y)+\int_{-\infty}^{0} \Omega_{-}(x+y+z) C(x, z) d z+\left[\begin{array}{c}
\Omega_{-}(x+y) \\
0
\end{array}\right]=0 .
$$

Proof. As $B_{y}(x, y)$ is integrable in $y, \xi g_{1}(x, \xi)$ is bounded. By (5.6), We can apply the Phragmén-Lindelöf type argument (see [9, Problem 325]) and conclude that, for $x>0, \zeta g_{1}(x, \zeta)$ is bounded in $\operatorname{Im} \zeta \geqq 0$. So $h(x, \zeta)$ $t(1,0) \rightarrow 0$ uniformly as $|\zeta| \rightarrow \infty, \operatorname{Im} \zeta \geqq 0$. By Jordan's lemma [12, §60],

$$
\int\left\{h(x, \xi){ }^{t}(1,0)\right\} \exp (2 i \xi y) d \xi=0, \quad y>0,
$$

holds. The first assertion follows.

By direct calculation, it is easy to show that

$$
-a(\xi)^{-1} h_{+}(x, \xi)=h^{\sharp}(x, \xi)+r_{-}(\xi) h(x, \xi) \exp (-2 i \xi x) .
$$

Taking into account (5.11) and repeating the argument of $\S 3$, the second assertion follows.

Q.E.D.

As the $L^{2}$-solution of the fundamental equation is uniquely determined (see the proof of Lemma 4.2),

$$
C(x, y)=B_{-}(x, y) \quad x>0
$$

i.e.

$$
\psi(x, \zeta)=\psi_{-}(x, \zeta) \quad x>0 .
$$

So we have (5.3) for $x>0$ and therefore $u_{ \pm}(x)$ coincide for $x>0$.

By the estimate (4.7), we finally have

Theorem 5.3. In addition to the assumption in the previous theorem, we assume that $m_{ \pm}( \pm x)$ are in $L^{1}(a, \infty)$. Then

$$
\left\{r_{ \pm}(\xi), \zeta_{j}, c_{j}^{ \pm}\right\}
$$

are the scattering data of an (uniquely determined) integrable potential which satisfies the assumptions of Theorem 1.4.

Application of Lemma 4.5 leads to 
Corollary 5.4. If $r_{+}(\xi)=r(\xi)$ belongs to $S$, then the potential belongs to $S$.

\section{§. Non-linear Schrödinger Equation}

We now turn to the construction of solutions for (0.1) in terms of scattering data of $L_{u}$.

Put

$$
B_{u}=\left[\begin{array}{rr}
1 & 0 \\
0 & -1
\end{array}\right]\left(D^{2}+2^{-1}|u|^{2}\right)-2^{-1}\left[\begin{array}{ll}
0 & u \\
u^{*} & 0
\end{array}\right] D-2^{-1} D\left[\begin{array}{ll}
0 & u \\
u^{*} & 0
\end{array}\right]
$$

Then the operator

$$
\left[B_{u}, L_{u}\right]=B_{u} L_{u}-L_{u} B_{u}
$$

is the multiplication by the matrix valued function:

$$
\left[B_{u}, L_{u}\right]=\left[\begin{array}{cc}
0 & -2^{-1} i u_{x x}-i|u|^{2} u \\
2^{-1} i u_{x x}^{*}+i|u|^{2} u^{*} & 0
\end{array}\right]
$$

So for $u=u(t)=u(x, t),(0.1)$ and the operator evolution equation

$$
d L_{u} / d t=i\left[B_{u}, L_{u}\right]
$$

are equivalent.

This relation is found in [15] and is different from the original relation between $L_{u}$ and (0.1) first given in [19]. A similar relation for the $\mathrm{KdV}$ equation has been found by Lax [7].

We now describe a procedure to determine the time variation of the Jost solutions and scattering data of $L_{u(t)}$ on a formal basis. Relations found here will be used later to construct solutions.

Differentiating $L_{u} \psi_{ \pm}=\zeta \psi_{ \pm}$with respect to $t$, we have

$$
\left(d L_{u} / d t\right) \psi_{ \pm}+L_{u}\left(d \psi_{ \pm} / d t\right)=\zeta d \psi_{ \pm} / d t
$$

If (6.2) holds, (6.3) can be written as

$$
L_{u}\left(d \psi_{ \pm} / d t-i B_{u} \psi_{ \pm}\right)=\zeta\left(d \psi_{ \pm} / d t-i B_{u} \psi_{ \pm}\right)
$$

Taking into account the asymptotic behavior and uniqueness of the Jost 
solutions, we obtain

$$
d \psi_{ \pm} / d t-i B_{u} \psi_{ \pm}=\mp i \zeta^{2} \psi_{ \pm}
$$

We proceed to the determination of the time variation of the scattering data. Differentiating the relation (2.1) with respect to $t$ and eliminating $d \psi_{ \pm} / d t$ by (6.4), we obtain an identity

$$
d a(\xi) / d t \psi_{+}^{\#}+\left(d b(\xi) / d t-2 i \xi^{2} b(\xi)\right) \psi_{+}=0 .
$$

So we have

$$
a(\xi, t)=a(\xi, 0), \quad b(\xi, t)=b(\xi, 0) \exp \left(2 i \xi^{2} t\right)
$$

$a(\zeta, t)$ is independent of $t$ and so are its zeros $\zeta_{1}, \ldots, \zeta_{N}$ in the upper half plane.

Differentiating (6.4) with respect to $\zeta$, we get

$$
\begin{aligned}
& d \psi_{ \pm}^{(k)} \mid d t-i B_{u} \psi_{ \pm}^{(k)} \\
& \quad=\mp i \zeta^{2} \psi_{ \pm}^{(k)} \mp 2 i k \zeta \psi_{ \pm}^{(k-1)} \mp i k(k-1) \psi_{ \pm}^{(k-2)} \quad\left(\zeta=\zeta_{0}\right)
\end{aligned}
$$

where $\psi^{(k)}=d^{k} \psi / d \zeta^{k}$. Then differentiate (2.11) in $t$ and eliminate $d \psi_{ \pm}^{(k)} / d t$ by (6.5). We have

$$
\begin{aligned}
& \sum_{\mu=0}^{k} \mu !^{-1}\left(d c_{k}^{ \pm}-\mu / d t \mp 2 i \zeta_{0}^{2} c_{k}^{ \pm}-\mu \mp 4 i \zeta_{0} c_{k-1-\mu}^{ \pm} \mp 2 i c_{k}^{ \pm}-2-\mu\right) \psi^{(\mu)}=0 \\
& \left(c_{-1}^{ \pm} \equiv c_{-2}^{ \pm} \equiv 0\right)
\end{aligned}
$$

From these relations, by induction on $k$, we obtain the system of differential equations for $c_{\frac{t}{k}}^{ \pm}(t)$ :

$$
d c_{k}^{ \pm} / d t= \pm\left(2 i \zeta_{0}^{2} c_{k}^{ \pm}+4 i \zeta_{0} c_{k-1}^{ \pm}+2 i c_{k}^{ \pm}-2\right), \quad 0 \leqq k \leqq m-1
$$

Next we consider the properties of differential equations (6.6). If these differential equations are satisfied, then $f_{ \pm}$defined by (3.4) satisfy

$$
\partial f_{ \pm} / \partial t=\mp 2^{-1} i \partial^{2} f_{ \pm} / \partial x^{2} .
$$

It is easy to show by induction that the solution $c_{k}^{ \pm}(t)$ are polynomials of order $k$ in $t$ multiplied by $\exp \left( \pm 2 i \zeta_{0}^{2} t\right)$. 
Lemma 6.1. If the relation (2.13) for $c_{\bar{k}}^{ \pm}(t)$ is satisfied at $t=0$, then it is satisfied for all $t$.

Proof. The $(i, j)$ element of the product on the left hand side of (2.13) is expressed as

$$
\sum_{k=j}^{m-i} c_{m-i-k}^{+}(t) c_{k-j}^{-}(t)
$$

Their t-derivatives are seen to vanish by (6.6).

Q.E.D.

Now we come to the construction of the solutions. Suppose $u(x)$ is an element of $S$. Then the coefficients $a(\zeta), b(\xi)$ and the scattering data $\left\{r_{ \pm}(\xi), \zeta_{j}, c_{j}^{ \pm}\right\}$of $L_{u}$ are determined. $a(\zeta)$ is assumed to satisfy our basic assumption (2.14). Let $\boldsymbol{c}_{j}^{ \pm}(t)$ be the chain of complex numbers obtained as the solution of (6.6) for the initial conditions $\boldsymbol{c}_{j}^{ \pm}$. Put $a(\zeta, t)=a(\zeta)$ and let $f_{ \pm j}(t)$ be defined by the chain $\boldsymbol{c}_{j}^{ \pm}(t)$ according to the formula (3.4) at the zero $\zeta=\zeta_{j}$ of $a(\zeta)$. Put

$$
r_{ \pm}(\xi, t)=r_{ \pm}(\xi) \exp \left( \pm 2 i \xi^{2} t\right)
$$

As $r_{ \pm}(\xi)$ are in $S$, so are $r_{ \pm}(\xi, t)$ in $\xi$

By Corollary 5.4, we have

Theorem 6.2. The collection

$$
\left\{r_{ \pm}(\xi, t), \zeta_{j}, c_{j}^{ \pm}(t)\right\}
$$

is the scattering data of $u(x, t)$ belonging to $S$ for each $t$.

If we prove that one of the relations (6.4) holds with the differentiability of $u(x, t)$ in $t$, then $u(x, t)$ is a solution of (0.1). In fact put (6.4) into $(6.3)$. Then

$$
\left(d L_{u} / d t-i\left[B_{u}, L_{u}\right]\right) \psi_{ \pm}=0
$$

follows. By $(6.1), u(x, t)$ is a solution of $(0.1)$. So we prove the equation $(6.4+)$ for $\psi_{+}$. We omit the suffix + in later discussion.

$(6.4+)$ is equivalent to $d h / d t=i g, h=h_{+}(x, \zeta ; t)$, where $g=g(x, \zeta ; t)$ is written as 


$$
\begin{aligned}
g= & {\left[\begin{array}{rr}
-2 & 0 \\
0 & 0
\end{array}\right] \zeta^{2} h+\left[\begin{array}{rr}
1 & 0 \\
0 & -1
\end{array}\right]\left(2 i \zeta h_{x}+h_{x x}+2^{-1}|u|^{2} h\right) } \\
& -\left[\begin{array}{ll}
0 & u \\
u^{*} & 0
\end{array}\right]\left(i \zeta h+h_{x}\right)-2^{-1}\left[\begin{array}{cc}
0 & u_{x} \\
u_{x}^{*} & 0
\end{array}\right] h .
\end{aligned}
$$

Put

$$
F(x, t)=\pi^{-1} \int r_{+}(\xi, t) \exp (2 i \xi x) d \xi
$$

and

$$
\Omega(x, t)=F(x, t)+\sum_{j=1}^{N} f_{j}(x, t) .
$$

The differential equation

$$
\Omega_{t}+2^{-1} i \Omega_{x x}=0
$$

holds as $F$ and $f_{j}$ satisfy the equation of the same form.

Let $B=B_{+}(x, y ; t)$ be the solution of the fundamental equation:

$$
B^{\sharp}(x, y ; t)+\int_{0}^{\infty} \Omega(x+y+z ; t) B(x, z ; t) d z+\left[\begin{array}{c}
0 \\
\Omega(x+y ; t)
\end{array}\right]=0 .
$$

The parameter $t$ will be omitted later for notational convenience.

By an argument analogous to Lemma 4.3 and the paragraph that follows, $B$ is differentiable in t. The equation

$$
B_{t}^{*}(x, y)+\int_{0}^{\infty} \Omega(x+y+z) B_{t}(x, z) d z+D(x, y)=0,
$$

where

$$
D(x, y)=-2^{-1} i \int_{0}^{\infty} \Omega_{x x}(x+y+z) B(x, z) d z-\left[\begin{array}{c}
0 \\
2^{-1} i \Omega_{x x}(x+y)
\end{array}\right],
$$

hold by (6.8). Using the relations (1.8), (1.9) and integrating by part, we have the following integral representation for $g$ defiıed by (6.7):

$$
g(x, \zeta ; t)=\int_{0}^{\infty} C(x, y ; t) \exp (2 i \zeta y) d y
$$

where 


$$
\begin{aligned}
& C_{1}(x, y)=2^{-1} B_{1 x x}(x, y)-u(x) B_{2 x}(x, y) \\
& C_{2}(x, y)=-2^{-1} B_{2 x x}(x, y)-u^{*}(x) B_{1 x}(x, y) .
\end{aligned}
$$

By direct calculation we have

$$
C \sharp(x, y)+\int_{0}^{\infty} \Omega(x+y+z) C(x, z) d z-i D(x, y)=0 .
$$

Comparing (6.9) and (6.10), we get

$$
B_{t}=i C
$$

because the homogeneous equation associated with the fundamental equation has only the trivial solution. The relation (6.4) has been established.

Thus we have proved

Theorem 6.3. The function $u(x, t)$ in the previous theorem satisfies $(0.1)$.

\section{§7. The Modified KdV Equation}

In this section we indicate how the real solution for the modified $\mathrm{KdV}$ equation (0.2) can be constructed.

Put

$$
A_{v}=-4 D^{3}+3\left[\begin{array}{cc}
-v^{2} & i v_{x} \\
i v_{x} & -v^{2}
\end{array}\right] D+3 D\left[\begin{array}{cc}
-v^{2} & i v_{x} \\
i v_{x} & -v^{2}
\end{array}\right]
$$

Then by direct calculation (0.2) is equivalent to

$$
d L_{i v} / d t=\left[A_{v}, L_{i v}\right]
$$

So it is necessary to find in terms of scattering data the condition that potential is purely-imaginary. Suppose that $u$ is purely imaginary. Then the Jost solution have the symmetry property

$$
\psi_{ \pm}\left(x,-\zeta^{*}\right)={ }^{t}\left(\mp \psi_{ \pm 1}^{*}(x, \zeta), \pm \psi_{ \pm 2}^{*}(x, \zeta)\right) .
$$

Then by (2.3),

$$
a\left(-\zeta^{*}\right)=a^{*}(\zeta), \quad b(-\xi)=-b^{*}(\xi)
$$


So the reflection coefficients satisfy

$$
r_{ \pm}(-\xi)=-r_{ \pm}^{*}(\xi)
$$

Concerning the zeros $\zeta_{j}(1 \leqq j \leqq N)$ of $a(\zeta)$, there exists a permutation $\sigma$ of order 2 of $\{1, \ldots, N\}$ (we can change the numbering of the zeros so that $\sigma(2 j-1)=2 j, \sigma(2 j)=2 j-1, j \leqq M ; \sigma(j)=j, j>2 M$ for certain integer $M$, $0 \leqq 2 M \leqq N$ ) such that

$$
\zeta_{\sigma(j)}=-\zeta_{j}^{*}
$$

and

$$
m(\sigma(j))=m(j)
$$

hold. The chain associated with the zero $\zeta_{j}$ should satisfy

$$
c_{\sigma(j), k}^{ \pm}=(-1)^{k+1} c_{j, k}^{ \pm *} \quad 0 \leqq k \leqq m(j)-1 .
$$

In fact, $(7.5+)$ follows from

$$
c^{*}\left(-\zeta^{*}\right)=-c(\zeta)
$$

in the notation of Theorem 2.3.

Lemma 7.1. If the basic assumption (2.14) is satisfied, conditions $(7.2+),(7.3),(7.4)$ and $(7.5+)$ are necessary and sufficient conditions for the potential to be purely imaginary valued.

Proof. Necessity has been already shown. Suppose these conditions are satisfied. Then we have

$$
\Omega_{+}^{*}(x)=-\Omega_{+}(x)
$$

So by the uniqueness of the solution of the fundamental equation

$$
B_{+i}^{*}(x, y)=(-1)^{i} B_{+i}(x, y) \quad i=1,2
$$

follow. Therefore the potential is purely imaginary valued. Q.E.D.

As the remaining argument is completely analogous to that of $\S 6$, we only indicate how the formulas should be modified.

If $v=v(t)=v(x, t)$ is a solution of (0.2) i.e. (7.1), then the Jost solutions 
should satisfy

$$
d \psi_{ \pm} / d t-B_{i v} \psi_{ \pm}= \pm 4 i \zeta^{3} \psi_{ \pm}
$$

So we have

$$
a(\zeta, t)=a(\zeta, 0), \quad b(\xi, t)=b(\xi, 0) \exp \left(8 i \xi^{3} t\right)
$$

and therefore

$$
r_{ \pm}(\xi, t)=r_{ \pm}(\xi, 0) \exp \left( \pm 8 i \xi^{3} t\right)
$$

(6.6) is replaced by

$$
d c_{k}^{ \pm} \mid d t= \pm\left(8 i \zeta_{0}^{3} c_{k}^{ \pm}+24 i \zeta_{0}^{2} c_{k}^{ \pm}-1+24 i \zeta_{0} c_{k}^{ \pm-2}+8 i c_{k-3}^{ \pm}\right), \quad 0 \leqq k \leqq m-1
$$

(7.6) is equivalent to $d h / d t=g, h=h_{+}$, where $g=g(x, \zeta ; t)$ is written as

$$
\begin{aligned}
& 12 \zeta^{2} h_{x}-12 i \zeta h_{x x}-4 h_{x x x} \\
& \quad+6\left[\begin{array}{cc}
-v^{2} & i v_{x} \\
i v_{x} & -v^{2}
\end{array}\right]\left(i \zeta h+h_{x}\right)+3\left[\begin{array}{cc}
-2 v v_{x} & i v_{x x} \\
i v_{x x} & -2 v v_{x}
\end{array}\right] h .
\end{aligned}
$$

We have the integral representation for $g$ :

$$
g(x, \zeta ; t)=\int_{0}^{\infty} C(x, y ; t) \exp (2 i \zeta y) d y
$$

where

$$
C=-B_{x x x}+3\left[\begin{array}{cc}
-v^{2} & i v_{x} \\
i v_{x} & -v^{2}
\end{array}\right] B_{x}
$$

The remaining part of argument is completely analogous to that of $\S 6$.

Our solutions contain all reflectionless solutions of (0.2). Previously a part of reflectionless solutions $(a(\zeta)$ with simple zeros) have been studied (Hirota [5], Tanaka [14], [15] and Wadati [17], [18]).

\section{References}

[1] Agranovich, Z. S. and Marchenko, V. A., The inverse problem of scattering theory, Kharkov Univ. Press 1960; English transl., Gordon \& Breach, New York 1963.

[2] Blashchak, V. A., Analogue of inverse scattering theory for non self-adjoint operator, Differential equation 4 (1968), 1519-1533, 1915-1924 (Russian). 
[3] Faddeev, L. D., Properties of the S-matrix of the one-dimensional Schrödinger equation, Proc. Steklov Inst. Math. 73 (1965), 314-336 (Russian).

[ 4 ] Gardner, O. S., Greene, J. M., Kruskal, M. D. and Miura, R. M., Method for solving the Korteweg-de Vries equations, Phys. Rev. Letters, 19 (1967), 1095-1097.

[5] Hirota, R., Exact solution of the modified Korteweg-de Vries equation for multiple collisions of solitons, J. Phys. Soc. Japan, 33 (1972), 1456-1458.

[6] Krein, M. G., Integral equations on a half line with kernel depending upon the difference of the arguments, Uspehi Mat. Nauk 13 (1958), 3-120 (Russian).

$\lfloor 7\rfloor$ Lax, P. D., Nonlinear partial differential equations of evolution, Actes Congres intern. Math., 1970. 2, 831-840.

[8] Marchenko, V. A., Spectral theory of Sturm-Liouville operators, Kiev, 1972 (Russian).

[9] Polya, G. and Szegö, G., Problems and Theorems in Analysis, Vol. 1, Springer-Verlag, Berlin-Heidelberg-New York 1972.

[10! Shabat, A. B., One dimensional scattering theory, Differential equations, 8 (1972), 164178 (Russian).

[11] Shabat, A. B., On a class of Wiener-Hopf type equations, D.A.N. 205 (1972), 546-549 (Russian).

[12] Smirnov, V. I., Complex variables-special functions, Vol. III-2 in A course of higher mathematics, Pergamon Press (1964).

[13] Tanaka, S., On the N-tuple wave solutions of the Korteweg-de Vries equation, Publ. RIMS, Kyoto Univ. 8 (1972/1973), 419-423.

[14] Tanaka, S., Some remarks on the modified Kortewg-de Vries equations, Publ. RIMS, Kyoto Univ. 8 (1972/1973), 429-437.

[15] Tanaka, S., Modified Korteweg-de Vries equation and scattering theory, Proc. Japan Acad. 48 (1972), 466-469.

[16] Tanaka, S., Analogue of Fourier's method for Korteweg-de Vries equation, Proc. Japan Acad. 48 (1972), 647-650.

[17] Wadati, M., The exact solution of the modified Korteweg-de Vries equation, J. Phys. Soc. Japan 32 (1972), 1981.

[18] Wadati, M., The modified Korteweg-de Vries equation, J. Phys. Soc. Japan 34 (1973), 1289-1296.

[19] Zakharov, V. E. and Shabat A. B., Exact theory of two-dimensional self-focussing and one-dimensional self-modulation of waves in nonlinear media, $J$. Exp. Theor. Phys. 61 (1971), 118-134 (Russian). 
